\title{
Institutional Quality and its Impact on FDI Inflow: Evidence from the EU Member States
}

\author{
Bogusława Dobrowolska (iD) https://orcid.org/0000-0003-3497-6223
}

Ph.D., University of Lodz, Lodz, Poland, e-mail: boguslawa.dobrowolska@uni.lodz.pl

Tomasz Dorożyński (iD https://orcid.org/0000-0003-3625-0354

Professor, University of Lodz, Lodz, Poland, e-mail: tomasz.dorozynski@uni.lodz.pl

Anetta Kuna-Marszałek (iD) https://orcid.org/0000-0001-5687-7272

Professor, University of Lodz, Lodz, Poland, e-mail: anetta.marszalek@uni.lodz.pl

\begin{abstract}
The aim of the article is to assess institutional quality in $28 \mathrm{EU}$ Member States and to examine the relationship between the quality of institutions and FDI inward stock as \% of GDP. This study is structured as follows. Firstly, we reviewed studies dedicated to the relationship between institutional quality and investment attractiveness. Then, we discussed FDI inflow into the EU countries and selected diagnostic variables that later served as the basis for our research in which we used categories of the Global Competitiveness Index. Based on rankings and using statistical methods, in the next stage, we divided the EU Member States into groups representing similar institutional quality. Then we investigated the relationships between groups of countries similar to one another when it comes to institutional quality and groups of countries ranked in ascending order by the value of foreign direct investment inflow measured as FDI inward stock as \% of GDP.

The study demonstrated that the EU Member States differ with respect to institutional quality. The results of the statistical analysis have provided grounds to positively verify the hypothesis about a positive relationship between the level of institutional quality and investment attractiveness.
\end{abstract}

Keywords: FDI, institutions, institutional quality, hierarchical cluster analysis, EU Member States

JEL: F21, F23 


\section{Introduction}

The answer to the question of why a country is an attractive investment destination for a company seems quite straightforward: firms choose the investment location that involves the highest expected profitability and reduces the riskiness and uncertainty of the investment. But what does it really mean? What exactly do the companies consider? The subject-matter literature highlights a number of different determinants of foreign direct investment (FDI) inflow. Their usual catalog includes the size and growth potential of the host market, economic stability, the degree of openness of the host economy, and income level, government spending, corporate tax rate, human capital and political stability, level of development, and the quality of institutions (Walsh, Yu 2010, p. 4; O’Meara 2015, p. 2; Chanegriha, Stewart, and Tsoukis 2017, pp. 759-776; Dellis, Sondermann, and Vansteenkiste 2017, p. 19). The last one is increasingly more often the main subject of research.

Scholarly interest in the institutional determinants of FDI coincides with the growing body of literature that has focused on governance and economic development (Buchanan, Le, and Rishi 2012, p. 81). It indicates, inter alia, that institutional quality (IQ) significantly differs for countries at different levels of economic development (Jabri, Brahim 2015, pp. 2001-2012; Bailey 2018, pp. 139-148). Usually, it is higher in developed economies than in emerging markets or developing countries. The subject-matter literature mostly claims that "a certain optimal level of institutional development is a prerequisite for the materialization of the growth-enhancing effect of FDI" (Yeboua 2020, p. 2) and that the host country's IQ “affects profitability, and institutionally strong countries can attract foreign investors by offering high returns" (Sabir et al. 2019, p. 4). In other words, countries in which IQ is higher can attract more capital, and poor-quality institutions hinder FDI inflow, acting like a tax and therefore are a cost to FDI (Buchanan, Le, and Rishi 2012, p. 82).

The principal goal of the article is to assess institutional quality in $28 \mathrm{EU}$ Member States and to examine the relationship between IQ and FDI inward stock as \% of GDP. To achieve this goal, we used various statistical methods, i.e., k-means clustering, hierarchical cluster analysis, contingency analysis, descriptive statistics, normality plots with tests, and M-estimators. The hypothesis states that there is a positive relationship between the level of institutional quality and investment attractiveness of EU countries measured with the value of FDI inward stock as \% of GDP.

This study is structured as follows. Firstly, we reviewed studies dedicated to the relationship between IQ and investment attractiveness. Then, we discussed FDI inflow into the EU countries and selected diagnostic variables that later served as the basis for our research in which we used categories of the Global Competitiveness Index. In the next stage, we divided the EU Member States into groups representing similar IQs. Then we investigated the relationships between groups of countries similar to one another when it comes to IQ and groups of countries ranked in ascending order by the value of FDI inflow measured as FDI inward stock as \% of GDP. 
The article sheds more light on the discussion about the relevance of IQ as a factor that determines FDI inflow. The added value of this article is derived from grouping $28 \mathrm{EU}$ Member States based on their similarity regarding IQ and demonstrating that it impacts the size of FDI inflow. We proposed an original set of indicators for these countries that help in identifying their IQ. The results from this study are useful for policymakers. Our findings make it possible to formulate policy implications that stress the role of IQ as an important determinant in attracting FDI inflows.

\section{Literature review}

The history of research studies conducted by economists and experts in management, international economics, and international business in the pursuit of understanding the factors that impact the location decisions of enterprises with foreign capital is a long-standing one. Nielsen, Asmussen, and Weatherall (2017, pp. 62-82) made an overview of 153 studies published in renowned scientific journals over the period 1976-2015 that were devoted to determinants of location choices made by MNEs. Their authors focused predominantly on the relationship between location choices and some attributes of the host country's economy, e.g., the size of its domestic market, taxes, salaries and wages, infrastructure, human capital resources, and the quality of the institutional framework. We decided to concentrate on the last one.

Institutions can be seen from diverse perspectives, as can the factors that impact their quality (Alonso and Garcimartín 2013, pp. 206-226; Kunčič 2014, pp. 135-161; Lehne, Mo, and Plekhanov 2014, pp. 1-22; Grabowski and Self 2020, pp. 1-12). The multiplicity of research approaches to the issue is stressed by Rodríguez-Pose (2013, p. 1037), who argued that "defining institutions is notoriously difficult and the current literature on the topic does not agree on a common definition". The same can be said about measuring IQ across different territorial contexts. For instance, in our analysis, we rely on the approach adopted by the World Economic Forum. Since 1979, it has published the Global Competitiveness Index (GCI), with IQ being one of its key pillars. As with any other such measures, these indicators are also imperfect and can be approached critically, but in our opinion, they represent the most comprehensive and measurable set of variables that capture IQ and are one of the best measures available.

The impact of institutional quality as a location factor that can stimulate or discourage FDI inflows was already indicated by Dunning (1980) in his eclectic paradigm. Later, many scholars dealt with these issues, most frequently demonstrating that better institutions in host countries help attract more FDI inflows (Tun, Azman-Saini, and Law 2012; Ullah, Khan 2017, pp. 1030-1050). According to Stachowiak (2007, p. 47), institutional differences between countries can be sources of comparative advantage because some activities are more institution-intensive than others, which may generate increased trade and capital flows. Multinational companies will be seeking to ex- 
ploit the above-mentioned comparative advantage of a given country by which they will contribute to the growth of FDI.

Researchers agree that institutions - be it regional, national, or even supranational - shape the nature of business by providing the framework of opportunities and constraints within which economic activity takes place (Nielsen, Asmussen, and Weatherall 2017, pp. 62-82), which implies that the quality of the host country's institutions should impact FDI location decisions. Thus, countries planning to attract more foreign capital should provide an appropriate institutional environment, e.g., in terms of political stability, property rights, or low levels of business uncertainty and risk. Moreover, according to many researchers, a higher IQ means that the consumer market of the country in question is more active and efficient, and consumer demand ensures the profitability of the investment projects carried out in this country (Aibai et al. 2019, p. 3278).

On the other hand, poor institutional quality can be an obstacle to FDI inflows, as it represents a threat to the investment and increases the cost of doing business (Aziz 2018, p. 111). Avoiding problems with regulatory, bureaucratic, and judicial hurdles, property rights, enforceable contracts, or performance and content requirements will be seen as positive by foreign investors; however, one needs to bear in mind that bribery and corruption are obviously more invasive in emerging markets than in developed economies (Dumludag 2009, p. 28).

While we can find in the subject-specific literature devoted to developing countries a broad consensus around the assertion that institutional quality is important to attract FDI, for developed countries, the claim is not so obvious (Dellis, Sondermann, and Vansteenkiste 2017, p. 6). Remarkably, researchers' attention is usually focused on developing and transitioning countries (Jabri, Brahim 2015, pp. 2001-2012), while developed economies are rarely examined in this context. In addition, the impact of IQ on FDI inflows is rarely investigated in parallel for developed and developing countries (Peres, Ameer, and Xu 2018, pp. 626-644).

The relationship between IQ and FDI inflows is illustrated in the literature by many factors, such as a political regime (Madani and Nobakht 2014, pp. 75-82; Moon 2019, pp. 1256-1277), corruption (Türedi 2018, pp. 151-172), tax policies (Ade, Rossouw, and Gwatidzo 2021, pp. 55-77), intellectual property rights (Hammami 2019, pp. 861871), quality of the educational system (Miningou and Tapsoba 2017), security (Essien et al. 2015), public and private sector transparency (Seyoum and Manyak 2009, pp. 187-206), government effectiveness (Sedik 2012), and even political parties (Bellinger and Son 2018, pp. 712-731). Most works reveal positive relationships between IQ and FDI; however, there are studies in which a set of institutional factors is statistically non-significant for attracting FDI flows, e.g., control of corruption and the rule of law (Bayar and Alakbarov 2016, pp. 303-308; Abdella, Naghavi, and Fah 2017, pp. 32-38), the quality of democracy, and political instability (Madr and Kouba 2015, pp. 2017-2026), government effectiveness (Jurčić, Franc, and Barišić 2020, pp. 44-57), or economic freedom (Subasat, Bellos 2011, pp. 2053-2065). 
Due to the multiplicity of factors that shape IQ, the literature on its impact upon FDI can be divided into three strands (Kurul and Yalta 2017, pp. 1-10). The first strand mainly focuses on identifying the effects of a specific institutional dimension, such as the influence of corruption or political regimes on FDI. The second strand analyzes the importance of different dimensions of IQ. Finally, the third strand explores the effect of a composite institutional indicator, which is constructed by combining different dimensions of institutional variables.

\section{The relationship between institutional quality and FDI inflow}

The principal goal of the study is to assess the relationship between the inflow of foreign direct investment and the institutional quality of countries. The study was based on statistical data for $28 \mathrm{EU}$ Member States. ${ }^{1}$ The empirical part consisted of three stages. In the first stage, based on the in-depth literature review, we selected variables that, to the best of our knowledge, best describe institutional quality. Partial indicators (20 variables) for 28 EU Member States were taken from the Global Competitiveness Index database. In the second stage, using statistical methods of cluster analysis (i.e., k-means clustering, hierarchical cluster analysis), we identified countries of similar institutional quality. Based on that, we divided the EU Member States into: (1) five groups of countries similar to one another regarding institutional quality and (2) four groups of countries ranked in ascending order by the value of foreign direct investment inflow measured as FDI inward stock as \% of GDP. In this case, we used UNCTAD data. In the last stage of the study, we investigated the relationships between them. The results allowed us to verify the hypothesis that there is a positive relationship between institutional quality and investment attractiveness of countries measured with the value of FDI inward stock as \% of GDP.

The European Union is often seen by investors as one entity, even though the Member States differ greatly from each other regarding their ability to attract FDI. The UK, the Netherlands, Germany, Ireland, and France are the most effective at successfully competing for foreign investors. In 2018, the total value of the FDI inward stock exceeded almost twice the value of FDI stock in all the other countries covered by the study taken together (UNCTAD 2019). However, the values look different when we consider the FDI inward stock as \% of GDP. Then, we see that Cyprus and Malta are the undisputed leaders (respectively, 1716\% and 1416\%). Ireland, Luxembourg, the Netherlands, and Belgium managed to attract FDI stock equivalent of more than $100 \%$ of their GDP. At the bottom of the ranking is Greece, with a share of FDI stock in GDP of ca. $16 \%$ (UNCTAD 2019). It is difficult to find regularities in this ranking that would address, e.g., the impact of the size of the country or EU membership experience.

1 Due to the period covered by the analysis (2018-2019), the UK is included in the study. 
As mentioned, in the first stage, we made a preliminary data analysis. The study was conducted for the years 2018-2019 based on 20 partial indicators selected from the Global Competitiveness Index (GCI) for 28 EU Member States (a detailed description of variables is given in the Appendix, Tab. A1). The structure of the study ensured comparability of values of individual indicators that, in addition, provided a detailed description of institutional quality in selected countries. The relatively short time horizon and, consequently, the inability to carry out a panel study was a clear disadvantage of the exercise. The timeframe limitation was forced by the fact that in 2017 , the GCI index methodology underwent many changes, meaning that results from before 2017 cannot be put together with indices for later years. Hence, the short time series determined the choice of statistical methods applied in the study.

Among the variables selected for the analysis, the following were the most differentiated: homicide rate, the efficiency of the legal framework in challenging regulations, and the efficiency of the legal framework to settle disputes. On the other hand, the least dispersion (statistically insignificant) amongst the EU Member States was observed for two variables: terrorism incidence and World Press Freedom Index. Most variables exhibited weak asymmetry, meaning that the frequency distributions around the mean were close to a symmetrical distribution. Thus, for the majority of variables (17), we were dealing with symmetrical distributions with little differentiation of results, and thus the use of the arithmetic mean to evaluate them can be considered correct at a substantive level (see Tab. 1). Exceptions were observed for only three variables characterized by very strong asymmetry and kurtosis of the distribution: homicide rate, terrorism incidence, and quality of land administration index.

Table 1. Descriptive statistics of institutional quality variables for 28 EU Member States in 2018-2019

\begin{tabular}{|l|c|c|c|c|c|c|}
\cline { 2 - 6 } \multicolumn{1}{l|}{} & Mean & $\begin{array}{c}\text { Std. } \\
\text { Deviation }\end{array}$ & Skewness & Kurtosis & Range $\begin{array}{c}\text { Coefficient } \\
\text { of variation } \\
\text { (\%) }\end{array}$ \\
\hline Organized crime (1-7) & 5.33 & 0.65 & -0.70 & 2.15 & 3.21 & 12.25 \\
\hline $\begin{array}{l}\text { Homicides per 100,000 } \\
\text { population }\end{array}$ & 1.35 & 1.08 & 2.68 & 8.08 & 4.97 & 80.12 \\
\hline $\begin{array}{l}\text { Terrorism incidence (0 = very high; } \\
\text { 100 = no incidence) }\end{array}$ & 97.00 & 9.55 & -4.76 & 23.81 & 50.00 & 9.85 \\
\hline Reliability of police services (1-7) & 5.17 & 0.84 & -0.16 & -0.74 & 3.31 & 16.29 \\
\hline Social capital (0-100, high) & 54.92 & 6.66 & -0.14 & -1.44 & 21.24 & 12.13 \\
\hline $\begin{array}{l}\text { Open Budget Data score } \\
\text { (0-100, best) }\end{array}$ & 62.09 & 17.36 & -1.33 & 3.57 & 84.68 & 27.96 \\
\hline Judicial independence (1-7) & 4.71 & 1.23 & -0.06 & -1.27 & 4.04 & 26.11 \\
\hline $\begin{array}{l}\text { Efficiency of legal framework } \\
\text { in challenging regulations (1-7) }\end{array}$ & 3.54 & 1.09 & 0.33 & -1.19 & 3.60 & 30.79 \\
\hline $\begin{array}{l}\text { World Press Freedom Index } \\
\text { (0 = most free; 100 = least free) }\end{array}$ & 80.38 & 6.87 & -0.34 & -0.57 & 26.83 & 8.55 \\
\hline $\begin{array}{l}\text { Burden of government regulation } \\
\text { (1-7) }\end{array}$ & 3.32 & 0.79 & 0.19 & -0.86 & 2.94 & 23.86 \\
\hline
\end{tabular}


Institutional Quality and its Impact on FDI Inflow: Evidence from the EU Member States

\begin{tabular}{|c|c|c|c|c|c|c|}
\hline $\begin{array}{l}\text { Efficiency of legal framework } \\
\text { to settle disputes (1-7) }\end{array}$ & 3.85 & 1.17 & 0.15 & -1.11 & 3.97 & 30.42 \\
\hline E-Participation Index (0-1, best) & 0.80 & 0.11 & -0.31 & -0.65 & 0.39 & 14.07 \\
\hline $\begin{array}{l}\text { Future orientation of government } \\
(1-7)\end{array}$ & 3.81 & 0.90 & 0.21 & -0.60 & 3.61 & 23.71 \\
\hline $\begin{array}{l}\text { Corruption Perception Index } \\
(0=\text { highly corrupt; } 100=\text { very } \\
\text { clean) }\end{array}$ & 64.55 & 14.09 & 0.19 & -1.38 & 45.00 & 21.83 \\
\hline Property rights (1-7) & 4.99 & 0.92 & 0.03 & -1.26 & 3.02 & 18.39 \\
\hline $\begin{array}{l}\text { Intellectual property protection } \\
(1-7)\end{array}$ & 5.05 & 0.87 & -0.09 & -1.06 & 3.22 & 17.25 \\
\hline $\begin{array}{l}\text { Quality of land administration } \\
\text { index }(0-30 \text {, best })\end{array}$ & 22.73 & 5.05 & -2.04 & 5.67 & 24.00 & 22.21 \\
\hline $\begin{array}{l}\text { Strength of auditing and account- } \\
\text { ing standards (1-7) }\end{array}$ & 5.20 & 0.75 & -0.04 & -1.08 & 2.75 & 14.42 \\
\hline $\begin{array}{l}\text { Extent of conflict of interest } \\
\text { regulation }(0-10, \text { best })\end{array}$ & 5.98 & 1.03 & 0.67 & 1.41 & 4.70 & 17.29 \\
\hline $\begin{array}{l}\text { Extent of shareholder governance } \\
(0-10 \text {, best) }\end{array}$ & 6.54 & 0.82 & -0.10 & -0.06 & 3.30 & 12.54 \\
\hline
\end{tabular}

Source: own elaboration based on the Global Competitiveness Index (GCI).

In the second stage of the analysis, using cluster analysis methods, we tried to select countries of similar institutional quality assessed based on 20 partial indicators included in Table 1. Using the k-means algorithm, ${ }^{2}$ we distinguished two clusters (Tab. 2).

Table 2. Allocation to clusters based on the k-means algorithm for the 28 EU Member States in 2018-2019

\begin{tabular}{|l|c|c|}
\hline \multicolumn{1}{|c|}{ Country } & Cluster & Distance \\
\hline Austria & 1 & 2.493 \\
\hline Belgium & 1 & 3.126 \\
\hline Bulgaria & 2 & 3.813 \\
\hline Croatia & 2 & 3.331 \\
\hline Cyprus & 2 & 2.609 \\
\hline The Czech Republic & 2 & 2.807 \\
\hline Denmark & 1 & 1.629 \\
\hline Estonia & 1 & 3.069 \\
\hline Finland & 1 & 3.358 \\
\hline France & 1 & 3.169 \\
\hline Germany & 1 & 2.478 \\
\hline Greece & 2 & 4.277 \\
\hline Hungary & 2 & 3.163 \\
\hline Ireland & 1 & 3.075 \\
\hline Italy & 2 & 3.532 \\
\hline Latvia & 2 & 2.597 \\
\hline
\end{tabular}

2 Find more: Tan, Steinbach, Kumar 2006, pp. 125-157. 
Table 2. (continued)

\begin{tabular}{|l|c|c|}
\hline \multicolumn{1}{|c|}{ Country } & Cluster & Distance \\
\hline Lithuania & 2 & 4.563 \\
\hline Luxembourg & 1 & 3.978 \\
\hline Malta & 2 & 5.104 \\
\hline The Netherlands & 1 & 2.533 \\
\hline Poland & 2 & 2.005 \\
\hline Portugal & 2 & 5.813 \\
\hline Romania & 2 & 1.830 \\
\hline Slovakia & 2 & 3.062 \\
\hline Slovenia & 2 & 2.671 \\
\hline Spain & 2 & 3.834 \\
\hline Sweden & 1 & 1.782 \\
\hline United Kingdom & 1 & 3.084 \\
\hline
\end{tabular}

Source: own elaboration (calculations made in PS IMAGO).

The first cluster consists of 12 countries, i.e., Austria, Belgium, Denmark, Estonia, Finland, France, Germany, Ireland, Luxembourg, the Netherlands, Sweden, and the United Kingdom. These are mainly the so-called 'old' EU Member States (with the exception of Estonia ${ }^{3}$ ), most of them situated in the northern part of the continent. The remaining sixteen EU Member States, i.e., mainly the so-called 'new' EU Member States and the countries of Southern Europe, are brought together in the second cluster.

Attention needs to be paid to the analysis of the basic descriptive characteristics presented in Table 3, which unambiguously shows that the descriptive statistics for countries of the first group of clusters, representing similar institutional quality, are higher for most variables. It means that their IQ is higher than that of countries from the second cluster.

Division into only two groups, however, is too general to carry out an in-depth analysis of the relationship between the IQ and investment attractiveness measured with the inflow of FDI. For this reason, in the next stage of the study, we deployed the hierarchical cluster analysis used to detect objects that are similar with regard to specific features in a set of data (Lasek 2002; James et al. 2014).

3 Estonia is the undisputed leader in institutional quality amongst the so-called 'new' EU Member States (see Dorożyński, Dobrowolska, and Kuna-Marszałek 2020, pp. 91-110). 
Institutional Quality and its Impact on FDI Inflow: Evidence from the EU Member States

\begin{tabular}{|c|c|c|c|c|c|c|c|c|c|c|c|c|c|c|c|c|c|c|}
\hline 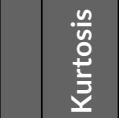 & $\stackrel{\sim}{\stackrel{\sim}{\rightarrow}}$ & 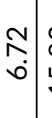 & 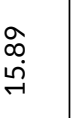 & $\begin{array}{c}\hat{\infty} \\
0 \\
0 \\
0\end{array}$ & $\begin{array}{l}0 \\
0 \\
0 \\
0\end{array}$ & & 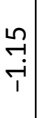 & 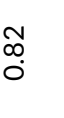 & 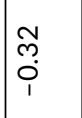 & $\mid \begin{array}{l}0 \\
0 \\
0\end{array}$ & 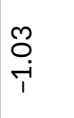 & $\left|\begin{array}{c}n \\
\hat{i} \\
1\end{array}\right|$ & $\begin{array}{l}\infty \\
\\
\rightarrow\end{array}$ & $\underset{\uparrow}{\stackrel{न}{\rightarrow}}$ & $\left|\begin{array}{l}0 \\
0 \\
i \\
1\end{array}\right|$ & 文 & $\begin{array}{l}\stackrel{\infty}{\leftrightarrow} \\
\stackrel{m}{m}\end{array}$ & o. \\
\hline 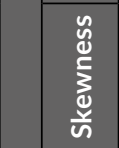 & $\begin{array}{l}0 \\
0 \\
+1\end{array}$ & 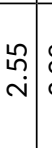 & 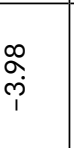 & 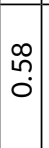 & 苚 & $\underset{\vec{p}}{\vec{p}}$ & 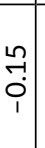 & $\begin{array}{l}\underbrace{t}_{0} \\
\text { i }\end{array}$ & \begin{tabular}{l}
8 \\
\multirow{2}{*}{} \\
$i$ \\
1
\end{tabular} & స̃ & $\begin{array}{l}0 \\
\text { g } \\
\text { in }\end{array}$ & 辛 & 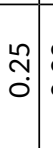 & $\begin{array}{c}\text { ָָ } \\
\text { ִָ }\end{array}$ & 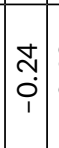 & $\mid \begin{array}{l}\infty \\
0 \\
0 \\
1 \\
1\end{array}$ & $\begin{array}{l}\overrightarrow{0} \\
\stackrel{i}{i}\end{array}$ & 苟 \\
\hline 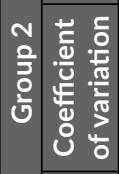 & $\stackrel{m}{0}$ & $\begin{array}{l}-\vec{a} \\
0\end{array}$ & $\stackrel{m}{\stackrel{m}{\sigma}}$ & 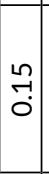 & $\vec{z}$ & હે & $\begin{array}{c}\infty \\
\stackrel{0}{0} \\
\vdots \\
0\end{array}$ & ఫे & O̊ & ने & ָָ & $\left|\begin{array}{c}n \\
\hdashline \\
0\end{array}\right|$ & $\begin{array}{c}\infty \\
\\
0\end{array}$ & ન્નુ & :-1 & \begin{tabular}{l}
-7 \\
\hdashline \\
0
\end{tabular} & $\stackrel{\substack{ָ \\
0}}{0}$ & न̈ \\
\hline के & . & 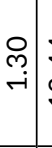 & 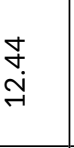 & $\begin{array}{l}a \\
0 \\
0 \\
0\end{array}$ & $\begin{array}{l}\text { So } \\
\text { in }\end{array}$ & 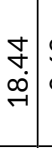 & $\begin{array}{l}a \\
\text { o. } \\
0 \\
0\end{array}$ & $\begin{array}{l}\text { חִ } \\
\text { nִ }\end{array}$ & $\begin{array}{l}\text { ô } \\
\text { in }\end{array}$ & {$\left[\begin{array}{l}n \\
n \\
0 \\
0\end{array}\right]$} & \begin{tabular}{l}
0 \\
\hdashline \\
0
\end{tabular} & $\begin{array}{l}-7 \\
0 \\
0\end{array}$ & $\begin{array}{l}n \\
0 \\
0\end{array}$ & $\begin{array}{l}\infty \\
0 \\
0 \\
0\end{array}$ & $\begin{array}{l}f \\
0\end{array}$ & 文 & $\begin{array}{l}8 \\
\text { o. }\end{array}$ & $\begin{array}{l}\text { ก̂? } \\
0\end{array}$ \\
\hline 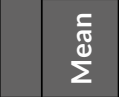 & $\begin{array}{l}0 \\
\text { ô }\end{array}$ & $\begin{array}{l}+ \\
+ \\
+\end{array}$ & 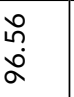 & 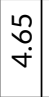 & $\begin{array}{c}0 \\
\text { aे } \\
\text { in }\end{array}$ & $\begin{array}{l}\hat{a} \\
\dot{\rho} \\
\hat{n}\end{array}$ & $\begin{array}{l}\stackrel{a}{a} \\
\dot{m}\end{array}$ & $\stackrel{\text { i }}{\mathrm{N}}$ & 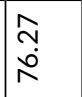 & $\begin{array}{l}\mid \begin{array}{l}\mid \\
\infty \\
i\end{array} \\
\end{array}$ & $\partial$ & \begin{tabular}{|l|}
\multirow{2}{*}{} \\
$\hat{0}$ \\
\end{tabular} & $\begin{array}{l}\text { ते } \\
\end{array}$ & $\begin{array}{l}\circ \\
\stackrel{\circ}{\circ} \\
\end{array}$ & $\begin{array}{l}\stackrel{\text { N }}{+} \\
\dot{f}\end{array}$ & \begin{tabular}{|c|} 
\\
$\vec{m}$ \\
$\dot{\sim}$
\end{tabular} & $\begin{array}{l}\underset{N}{N} \\
\dot{N}\end{array}$ & 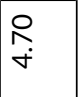 \\
\hline $\begin{array}{l}\frac{n}{4} \\
\frac{0}{3} \\
\frac{0}{3}\end{array}$ & $\underset{\overbrace{}}{\stackrel{N}{i}}$ & $\begin{array}{l}\vec{f} \\
\dot{\sim}\end{array}$ & $\underset{\sim}{\widehat{્}}$ & 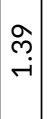 & $\begin{array}{l}\stackrel{n}{n} \\
\rightarrow\end{array}$ & $\begin{array}{l}0 \\
\dot{m} \\
\dot{m}\end{array}$ & 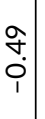 & & $\begin{array}{l}a \\
\hat{0}\end{array}$ & $\mid \begin{array}{l}\vec{f} \\
0 \\
1 \\
1\end{array}$ & $\underset{\rightarrow}{\stackrel{\infty}{\rightarrow}}$ & $\mid \begin{array}{l}0 \\
\hat{u} \\
0\end{array}$ & 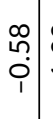 & 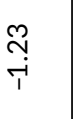 & $\mid \begin{array}{c}\tilde{n} \\
\tilde{1} \\
0 \\
1\end{array}$ & 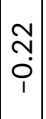 & $\underset{7}{\stackrel{7}{7}}$ & 吕 \\
\hline 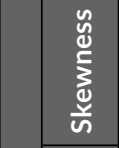 & $\stackrel{\leftrightarrow}{\rightarrow}$ & 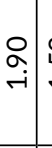 & רָ & $\begin{array}{c}\hat{N} \\
\hat{0}\end{array}$ & \begin{tabular}{c}
\multirow{J}{*}{} \\
$\stackrel{i}{i}$
\end{tabular} & $\begin{array}{c}\text { m} \\
\rightarrow \\
\rightarrow\end{array}$ & ָָ & ‡̛. & 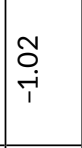 & $\mid \begin{array}{c}n \\
0 \\
0 \\
0 \\
1\end{array}$ & $\begin{array}{l}\text { No } \\
\text { î. }\end{array}$ & 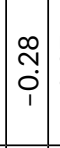 & \begin{tabular}{l}
\multirow{2}{0}{} \\
.
\end{tabular} & 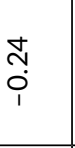 & 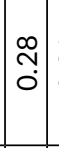 & స్ & ồ & $\begin{array}{l}0 \\
\stackrel{0}{i} \\
1\end{array}$ \\
\hline 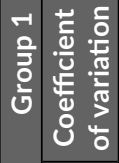 & . & 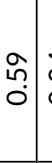 & $\begin{array}{l}\text { O. } \\
\text { O. }\end{array}$ & $\begin{array}{l}0 \\
0 \\
0\end{array}$ & ô. & ণৃ. & $\begin{array}{l}\hat{0} \\
0\end{array}$ & 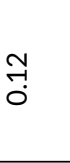 & 贻 & \begin{tabular}{l}
$n$ \\
\hdashline \\
0
\end{tabular} & సี & $\mid \begin{array}{l}0 \\
0 \\
0 \\
0\end{array}$ & \begin{tabular}{l}
7 \\
\hdashline \\
0
\end{tabular} & $\begin{array}{l}\text { ô. } \\
\text { o. }\end{array}$ & $\mid \begin{array}{l}0 \\
0 \\
0\end{array}$ & $\mid \begin{array}{l}0 \\
0 \\
0 \\
0\end{array}$ & 웅 & 恶 \\
\hline के & : & 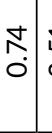 & 点 & $\mid \begin{array}{l}7 \\
\tilde{0}\end{array}$ & $\begin{array}{l}\stackrel{8}{\circ} \\
\stackrel{+}{*}\end{array}$ & 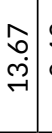 & 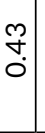 & 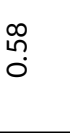 & $\underset{\stackrel{\rho}{\sim}}{\stackrel{\rho}{+}}$ & $\mid \begin{array}{l}-1 \\
0 \\
0\end{array}$ & $\begin{array}{l}\text { ôn } \\
\text { in }\end{array}$ & $\mid \begin{array}{l}\infty \\
0 \\
0\end{array}$ & \begin{tabular}{c}
$\infty$ \\
$\stackrel{2}{2}$ \\
\hdashline
\end{tabular} & $\begin{array}{l}\text { d } \\
\text { o } \\
\text { in }\end{array}$ & $\mid \begin{array}{c}\tilde{J} \\
\tilde{j} \\
0\end{array}$ & $\mid \begin{array}{c}0 \\
0 \\
0 \\
0\end{array}$ & ํㅗㄴ & $\begin{array}{l}\infty \\
\dddot{m} \\
0\end{array}$ \\
\hline 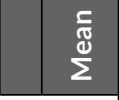 & 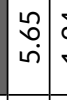 & 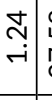 & 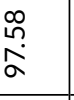 & \begin{tabular}{|l}
0 \\
$\infty$ \\
in
\end{tabular} & $\begin{array}{l}m \\
0 \\
0 \\
0\end{array}$ & $\begin{array}{l}-1 \\
0 \\
0 \\
0\end{array}$ & $\begin{array}{c}\text { वे } \\
\text { in }\end{array}$ & $\begin{array}{l}\text { ָे } \\
\stackrel{+}{*}\end{array}$ & $\begin{array}{l}\infty \\
\infty \\
\omega \\
\infty \\
\infty\end{array}$ & $\mid \begin{array}{l}0 \\
2 \\
\end{array}$ & ㅇ. & $\mid \begin{array}{l}0 \\
0 \\
0\end{array}$ & $\begin{array}{l}\mathbb{N} \\
\dot{+}\end{array}$ & 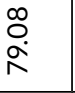 & $\left|\begin{array}{l}-1 \\
0 \\
i\end{array}\right|$ & 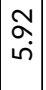 & $\begin{array}{l}\stackrel{n}{\sim} \\
\stackrel{\sim}{\sim}\end{array}$ & {$\left[\begin{array}{l}\infty \\
\infty \\
\infty \\
i n\end{array}\right.$} \\
\hline & 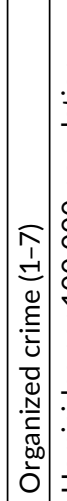 & 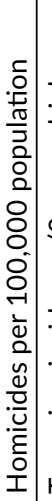 & 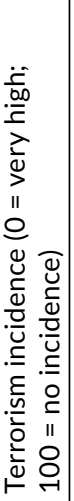 & 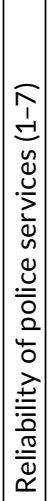 & 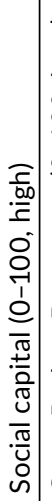 & 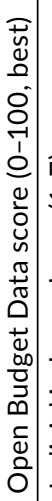 & & 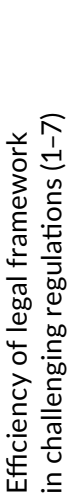 & 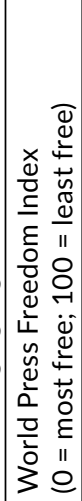 & 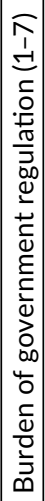 & 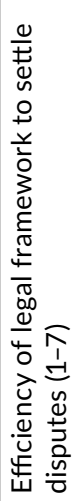 & 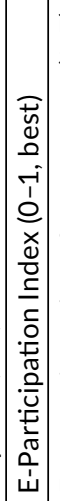 & 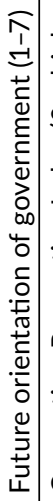 & 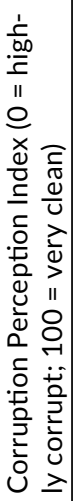 & 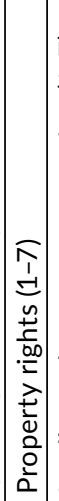 & 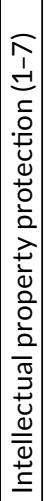 & 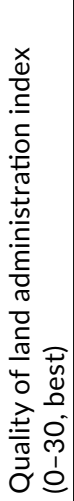 & 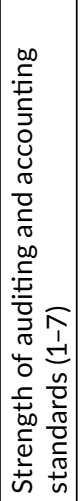 \\
\hline
\end{tabular}


Bogusława Dobrowolska, Tomasz Dorożyński, Anetta Kuna-Marszałek

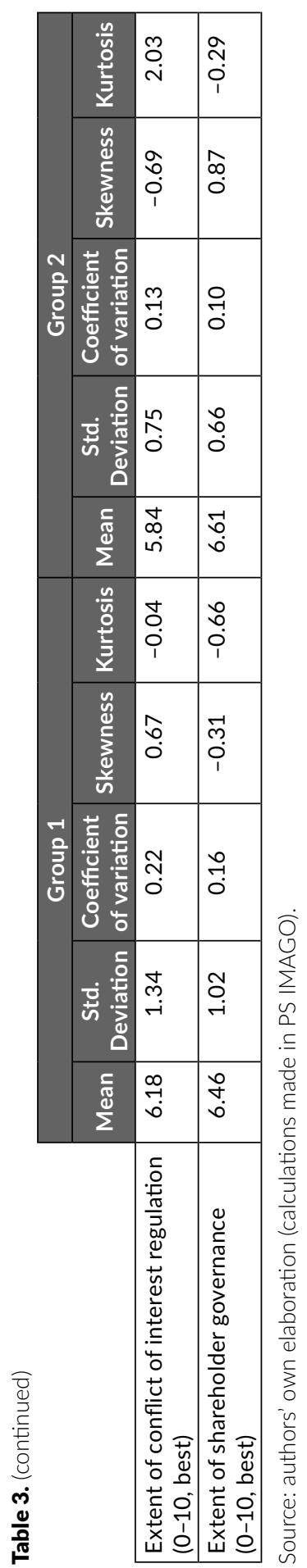




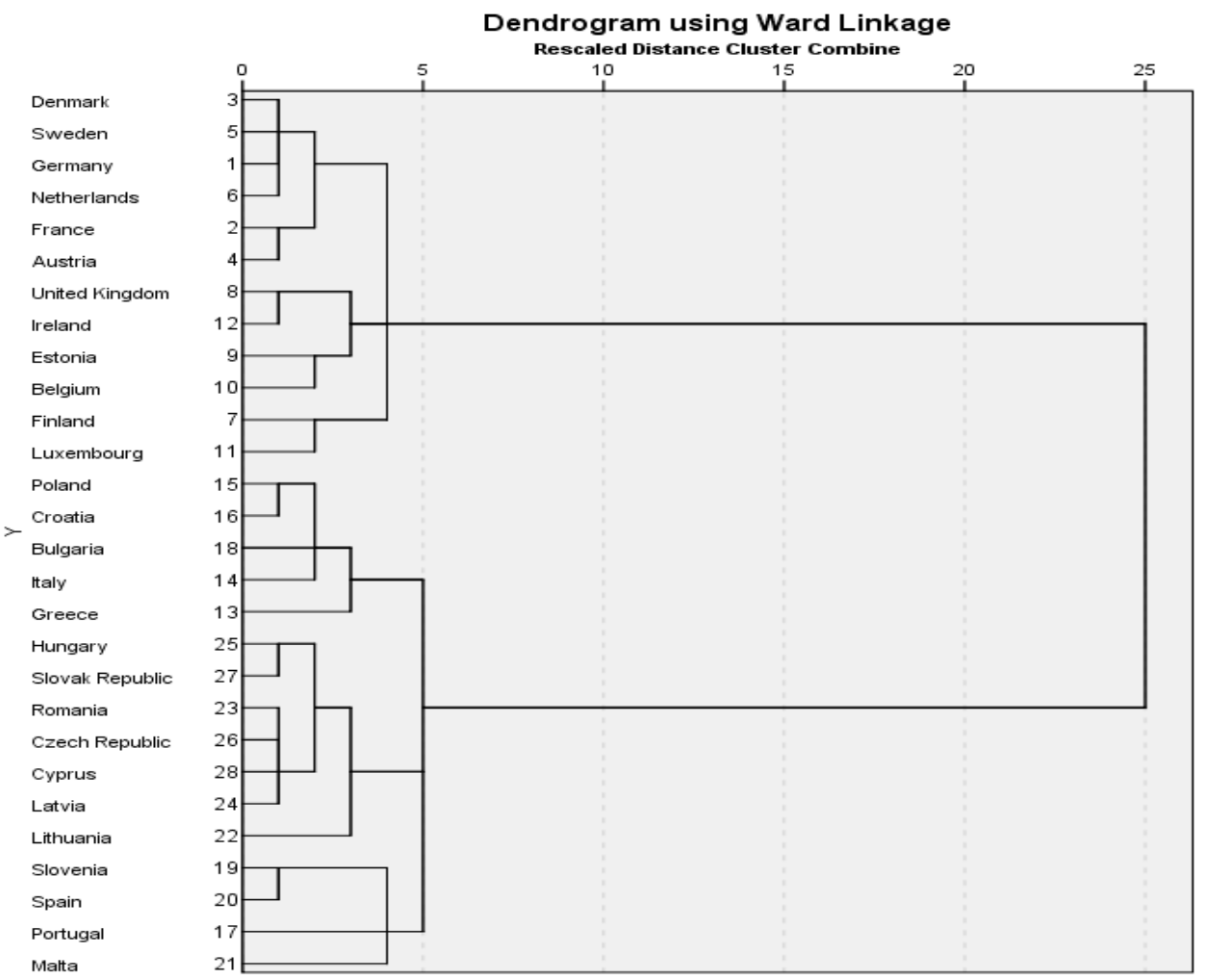

Figure 1. Dendrogram for 28 EU Member States obtained using the Ward method (Ward's linkage) Source: own elaboration.

Using the Ward method, we obtained a dendrogram revealing a hierarchical structure of a set of objects sorted from the most to the least similar. Thus, we were able to distinguish two principal clusters of the EU Member States that differ in institutional quality and, at the same time, divide the population covered by the study in a more detailed way. Thus, out of the investigated set of 28 EU Member States, we distinguished the five following groups (Figure 1):

- group 1: Denmark, Sweden, Austria, France, Germany, the Netherlands,

- group 2: Belgium, Estonia, Finland, Ireland, Luxembourg, United Kingdom,

- group 3: Croatia, Poland, Bulgaria, Italy, Greece, Portugal,

- group 4: Slovenia, Spain, Malta,

- group 5: Hungary, Slovakia, the Czech Republic, Romania, Cyprus, Latvia, Lithuania.

To compare IQ within the above-distinguished groups, we created a ranking based on the arithmetic mean for each of the 20 partial variables (see Appendix, Tab. A2), where the best value from the point of view of the evaluation of IQ was given 5 and the worst, 1 . Next, the results were corrected using weights assigned to each variable in the overall evaluation of the 'institutional quality' pillar of the GCI index. Nota- 
bly, the ranking of 20 variables that describe IQ in the five distinguished groups given in Table 4 clearly indicates that countries from groups 1 and 2 exhibit the highest institutional quality. Hence, the leaders are Denmark, Sweden, Austria, France, Germany, the Netherlands, Belgium, Estonia, Finland, Ireland, Luxembourg, and the United Kingdom. They are followed by countries from groups 4 and 5. Members of group 3, i.e., Croatia, Poland, Bulgaria, Italy, Greece, and Portugal, are at the bottom of the ranking, and their performance was assessed as the poorest in 13 out of 20 indicators.

Table 4. Ranking of institutional quality variables of the EU Member States in the years 2018-2019 by groups distinguished based on the dendrogram

\begin{tabular}{|l|c|c|c|c|c|}
\hline \multicolumn{1}{|c|}{ Country Group (1-5_) } & Group 1 & Group 2 & Group 3 & Group 4 & Group 5 \\
\hline Organized crime (1-7) & 9.375 & 15.625 & 3.125 & 12.5 & 6.25 \\
\hline Homicides per 100,000 population & 9.375 & 12.5 & 6.25 & 15.625 & 3.125 \\
\hline $\begin{array}{l}\text { Terrorism incidence (0 = very high; } \\
100=\text { no incidence) }\end{array}$ & 6.25 & 9.375 & 3.125 & 12.5 & 15.625 \\
\hline Reliability of police services (1-7) & 12.5 & 15.625 & 3.125 & 9.375 & 6.25 \\
\hline Social capital (0-100, high) & 62.5 & 37.5 & 25 & 50 & 12.5 \\
\hline Open Budget Data score (0-100, best) & 15.625 & 12.5 & 6.25 & 3.125 & 9.375 \\
\hline Judicial independence (1-7) & 12.5 & 15.625 & 3.125 & 9.375 & 6.25 \\
\hline $\begin{array}{l}\text { Efficiency of legal framework } \\
\text { in challenging regulations (1-7) }\end{array}$ & 12.5 & 15.625 & 3.125 & 9.375 & 6.25 \\
\hline $\begin{array}{l}\text { World Press Freedom Index (0 = most } \\
\text { free; 100 = least free) }\end{array}$ & 3.125 & 6.25 & 15.625 & 12.5 & 9.375 \\
\hline Burden of government regulation (1-7) & 16.64 & 20.8 & 4.16 & 12.48 & 8.32 \\
\hline $\begin{array}{l}\text { Efficiency of legal framework to settle } \\
\text { disputes (1-7) }\end{array}$ & 20.8 & 16.64 & 4.16 & 8.32 & 12.48 \\
\hline E-Participation Index (0-1, best) & 20.8 & 14.56 & 8.32 & 14.56 & 4.16 \\
\hline Future orientation of government (1-7) & 50 & 62.5 & 12.5 & 37.5 & 25 \\
\hline $\begin{array}{l}\text { Corruption Perception Index (0 = highly } \\
\text { corrupt; 100 = very clean) }\end{array}$ & 62.5 & 50 & 12.5 & 37.5 & 25 \\
\hline Property rights (1-7) & 16.64 & 20.8 & 4.16 & 12.48 & 8.32 \\
\hline Intellectual property protection (1-7) & 16.64 & 20.8 & 4.16 & 12.48 & 8.32 \\
\hline $\begin{array}{l}\text { Quality of land administration index } \\
\text { (0-30, best) }\end{array}$ & 20.8 & 16.64 & 4.16 & 8.32 & 12.48 \\
\hline $\begin{array}{l}\text { Strength of auditing and accounting } \\
\text { standards (1-7) }\end{array}$ & 20.8 & 16.64 & 4.16 & 12.48 & 8.32 \\
\hline $\begin{array}{l}\text { Extent of conflict of interest regulation } \\
\text { (0-10, best) }\end{array}$ & 8.32 & 20.8 & 12.48 & 16.64 & 4.16 \\
\hline $\begin{array}{l}\text { Extent of shareholder governance } \\
\text { (0-10, best) }\end{array}$ & 20.8 & 4.16 & 12.48 & 16.64 & 8.32 \\
\hline Total points & 418.49 & 404.97 & 151.99 & 323.78 & 199.88 \\
\hline Source: own elat & & & & & \\
\hline
\end{tabular}

Source: own elaboration (calculations made in PS IMAGO).

In the third stage, having grouped countries into clusters of the most similar institutional quality, we were able to find out about their cointegration with the inflow of FDI measured as FDI inward stock as \% of GDP. To this end, we transformed the 
FDI as \% of GDP variable measured on a quantitative measurement scale into a variable measured on an ordinal scale due to the clear deviation of this variable distribution from the normal distribution as suggested by both the results obtained from the Shapiro-Wilk ${ }^{4}$ test and the Q-Q plot with a trend.

Deviation in the distribution of the FDI inward stock as \% of GDP variable from the normal distribution is also confirmed by the descriptive statistics, in particular, by the $5 \%$ trimmed mean, the confidence interval for the mean, the skewness ratio, kurtosis, and the M-Estimators (see Tab. 5). Under the absence of homogeneity in this collection, we divided the EU Member States into four groups ranked by the FDI inward stock as \% of GDP variable in ascending order based on position metrics, such as quartiles.

Table 5. Statistics describing FDI inward stock as \% of GDP in the EU Member States in the years 2018-2019

\begin{tabular}{|l|c|c|}
\hline \multicolumn{1}{|c|}{ Descriptives } & Statistics & Std. Error \\
\hline Mean & 130.0079 & 59.83729 \\
\hline 95\% Confidence Interval for Mean - Lower Bound & 7.2319 & \\
\hline 95\% Confidence Interval for Mean - Upper Bound & 252.7838 & \\
\hline 5\% Trimmed Mean & 71.2374 & \\
\hline Variance & 100254.038 & \\
\hline Std. Deviation & 316.62918 & \\
\hline Minimum & 15.98 & \\
\hline Maximum & 1716.27 & \\
\hline Range & 1700.29 & \\
\hline Interquartile Range & 46.40 & \\
\hline Skewness & 5.001 & .441 \\
\hline Kurtosis & 25.789 & .858 \\
\hline Percentiles 25 & 33.2025 & \\
\hline Percentiles 50 & 52.9050 & \\
\hline Percentiles 75 & 79.6050 & \\
\hline \multicolumn{2}{|c|}{ M-Estimators } \\
\hline Huber's M-Estimator ${ }^{\mathrm{a}}$ & 54.9188 & \\
\hline Tukey's Biweight ${ }^{\mathrm{b}}$ & 47.6765 & \\
\hline Hampel's M-Estimator ${ }^{\mathrm{c}}$ & 50.3650 & \\
\hline Andrews' Wave ${ }^{\mathrm{d}}$ & 47.5541 & \\
\hline
\end{tabular}

a The weighting constant is 1.339 .

${ }^{b}$ The weighting constant is 4.685 .

c The weighting constants are $1.700,3.400$, and 8.500

d The weighting constants are $1.700,3.400$, and 8.500

Source: own elaboration (calculations made in PS IMAGO).

4 Statistics in the Shapiro - Wilk test is $0.321 ; \mathrm{df}=28, \mathrm{p}=0.0001$. 
The groups of countries distinguished by the value of FDI based on quartiles and the distribution by groups of institutional quality of $28 \mathrm{EU}$ Member States allowed us to create a cross table (Tab. 6). From the table, we can learn that countries representing higher institutional quality usually attract more FDI inward stock as \% of GDP.

Table 6. EU Member States allocation to groups of institutional quality and FDI inward stock as \% of GDP

\begin{tabular}{|l|c|c|c|c|c|c|}
\hline & \multicolumn{5}{|c|}{ Institutional quality groups } \\
\cline { 2 - 6 } $\begin{array}{l}\text { FDI inward stock } \\
\text { as \% of GDP }\end{array}$ & $\begin{array}{c}\text { Group 1 } \\
\text { DK, SE, AT, } \\
\text { FR, DE, NL }\end{array}$ & $\begin{array}{c}\text { Group 2 } \\
\text { BE, EE, FI, } \\
\text { IE, LU, UK }\end{array}$ & $\begin{array}{c}\text { Group 3 } \\
\text { HR, PL, } \\
\text { BG, IT, } \\
\text { EL, PT }\end{array}$ & $\begin{array}{c}\text { Group 4 } \\
\text { SI, ES, MT }\end{array}$ & $\begin{array}{c}\text { Group 5 } \\
\text { HU, SK, CZ, } \\
\text { RO, CY, LV, LT }\end{array}$ & Total \\
\hline $\begin{array}{l}\text { Group 1 } \\
\text { CY, MT, IE, LU, NL, } \\
\text { BE, EE }\end{array}$ & 1 & 4 & 0 & 1 & 1 & 7 \\
\hline $\begin{array}{l}\text { Group 2 } \\
\text { HU, SE, PT, CZ, UK, } \\
\text { BG }\end{array}$ & 1 & 1 & 2 & 0 & 2 & 6 \\
\hline $\begin{array}{l}\text { Group 3 } \\
\text { LT, RO, PL, AT, HR, } \\
\text { LV, ES, SK }\end{array}$ & 1 & 0 & 2 & 1 & 4 & 8 \\
\hline $\begin{array}{l}\text { Group 4 } \\
\text { EL, IT, DE, FI, FF, } \\
\text { DK, SI }\end{array}$ & 3 & 1 & 2 & 1 & 0 & 7 \\
\hline \begin{tabular}{l} 
Total \\
\hline
\end{tabular} & 6 & 6 & 6 & 3 & 7 & 28 \\
\hline
\end{tabular}

Source: own elaboration.

To assess the strength of the correlation between the institutional quality category of a country and the value of FDI inflow, we used the contingency coefficient. For the examined sample, the coefficient amounts to $0.595 .{ }^{5}$ It means that there is a significant positive correlation ${ }^{6}$ between the institutional quality category of a country and FDI inward stock as \% of GDP. The results of the above statistical analysis have provided grounds to positively verify the hypothesis about a positive relationship between the level of institutional quality and the investment attractiveness of countries measured with the value of FDI inward stock as \% of GDP.

5 Own calculations made using PS IMAGO.

6 The direction of the relationship was assessed based on the distribution of data in the contingency table (Table 6) and the ranking of variables describing institutional quality of the EU Member States (Table 4). 


\section{Conclusion}

The principal scientific goal of the study was to evaluate the relationship between the inflow of foreign direct investment and the institutional quality of countries. As mentioned above, there is a long list of location-specific attributes that impact foreign direct investment inflow. The dominant view is that countries with better institutional quality can attract more FDI. Poor quality institutions hinder FDI inflow, acting as a barrier to foreign investors unwilling to invest in countries where institutions encourage, e.g., corruption or nepotism. However, there are researchers who did not observe any clear relationship between institutional performance and FDI inflow. Ambiguous and sometimes even contradictory conclusions are, in our opinion, the fundamental reason why in-depth studies on the role of institutions in attracting foreign investors should be conducted.

In our case, the institutional performance of $28 \mathrm{EU}$ Member States was assessed based on the Global Competitiveness Index. Measuring institutional quality remains problematic for many researchers as there is no coherent measure. This is why we tried to develop our own measure, built of 20 partial variables from the first pillar of the GCI 'Institutions'. All of the selected indicators help assess the institutional system of a given country from the point of view of, inter alia, institutions' interference in the economy, and the efficiency, transparency, and quality of administration.

In the group of countries covered by the study, Finland is the undisputed leader when it comes to the quality of institutions. Simultaneously, it turned out that the examined countries can be divided into five groups representing similar institutional quality. Using the hierarchical cluster analysis, we selected five such groups. The institutional quality leaders among the 28 EU Member States are Denmark, Sweden, Austria, France, Germany, the Netherlands, Belgium, Estonia, Finland, Ireland, Luxembourg, and the United Kingdom. The group of the institutional "outsiders" is represented by both the 'old' and 'new' EU Member States, i.e., Croatia, Poland, Bulgaria, Italy, Greece, and Portugal. Poland is the only economy that is not in Southern Europe.

Surprisingly, there is a relatively big difference between the leaders of institutional quality and the group of institutional 'outsiders' in the ranking. The scores for countries from groups 1 and 2 are almost three times higher than those for group 3 (see Tab. 4). Groups 1 and 2 consist almost exclusively of the 'old' EU Member States. Estonia, the leader amongst countries of Central and Eastern Europe in institutional quality rankings, is the only exception here (see Dorożyński, Dobrowolska, and Kuna-Marszałek 2020, pp. 91-110).

When it comes to FDI inward stock as \% of GDP, we have found that Cyprus and Malta are at the top of the ranking, followed by Ireland, Luxembourg, the Netherlands, and Belgium, which managed to attract FDI stock equivalent to more than $100 \%$ of their GDP. Using various statistical methods, we examined the relationship between institutional quality and FDI inflow. It turned out that there is a statistically 
significant relationship between the level of institutional quality and investment attractiveness of countries measured with the value of FDI inward stock as \% of GDP. This means that relatively poor institutional quality may have a negative impact on the inflow of FDI.

The study has implications for research and practice. The results will be of interest to policymakers and may have an application value for institutions. An efficient and effective institutional system may importantly contribute to boosting the investment attractiveness of countries and impact FDI flows.

The limitation of the study is its relatively short time horizon and, consequently, the inability to deploy more sophisticated quantitative instruments and measures. Hence, the short time series determined the choice of statistical methods applied in the study. The future research agenda should focus on solving the problem of limited data in order to be able to employ, e.g., panel data analysis.

\section{References}

Abdella, A.B., Naghavi, N., Fah, B.C. (2017), The Effect of Corruption, Trade Openness and Political Stability on Foreign Direct Investment: Empirical Evidence from BRIC Countries, "International Journal of Advanced and Applied Sciences", 5 (3), pp. 3238, https://doi.org/10.21833/ijaas.2018.03.005

Ade, M., Rossouw, J., Gwatidzo, T. (2021), The effect of tax harmonization in the Southern African Development Community on foreign direct investment, "Journal of Developing Areas”, 55 (1), https://doi.org/10.1353/jda.2021.0006

Aibai, A., Huang, X., Luo, Y., Peng, Y. (2019), Foreign Direct Investment, Institutional Quality and Financial Development along the Belt and Road: An Empirical Investigation, "Emerging Markets Finance and Trade", 55 (14), pp. 3275-3294, https://doi .org/10.1080/1540496X.2018.1559139

Alonso, J.A., Garcimartín, C. (2013), The Determinants of Institutional Quality. More on the Debate, "Journal of International Development", 25 (2), pp. 206-226, https:// doi.org/10.1002/jid.1710

Aziz, O.G. (2018), Institutional quality and FDI inflows in Arab economies, "Finance Research Letters", 25, pp. 111-123, https://doi.org/10.1016/j.frl.2017.10.026

Bailey, N. (2018), Exploring the relationship between institutional factors and FDI attractiveness: A meta-analytic review, "International Business Review", 27 (1), pp. 139148, https://doi.org/10.1016/j.ibusrev.2017.05.012

Bayar, Y., Alakbarov, N. (2016), Corruption and Foreign Direct Investment Inflows in Emerging Market Economies, "Ecoforum Journal", 5 (2), pp. 303-308.

Bellinger, N.M., Son, B. (2018), Political Parties and Foreign Direct Investment Inflows among Developing Countries, "Political Studies”, 67 (3), pp. 712-731, https://doi.org /10.1177/0032321718799015

Buchanan, B.G., Le, Q.V., Rishi, M. (2012), Foreign direct investment and institutional quality: Some empirical evidence, "International Review of Financial Analysis", 21 (6), pp. 81-89, https://doi.org/10.1016/j.irfa.2011.10.001 
Chanegriha, M., Stewart, C., Tsoukis, C. (2017), Identifying the robust economic, geographical and political determinants of FDI: an Extreme Bounds Analysis, "Empirical Economics", 52 (2), pp. 759-776, https://doi.org/10.1007/s00181-016-1097-1

Dellis, K., Sondermann, D., Vansteenkiste, I. (2017), Determinants of FDI inflows in advanced economies: Does the quality of economic structures matter?, "ECB Working Paper", No. 2066.

Dorożyński, T., Dobrowolska, B., Kuna-Marszałek, A. (2020), Institutional Quality in Central and East European Countries and Its Impact on FDI Inflow, "Entrepreneurial Business and Economics Review", 8 (1), pp. 91-110, https://doi.org/10.156 78/EBER.2020.080105

Dumludag, D. (2009), An analysis of the determinants of foreign direct investment in Turkey: The role of the institutional context, "Journal of Business Economics and Management”, 10 (1), pp. 15-30, https://doi.org/10.3846/1611-1699.2009.10.15-30

Dunning, J.H. (1980), Toward an Eclectic Theory of International Production: Some Empirical Tests, "Journal of International Business Studies”, 11 (1), pp. 9-31. https:// doi.org/10.1057/palgrave.jibs.8490593

Essien, J., Barikui, T., Abuba, S., Igbara, F. (2015), The Impact of National Security on Foreign Direct Investment (FDI) in Nigeria, "IOSR Journal of Business and Management", 17 (5), pp. 69-74.

Grabowski, R., Self, S. (2020), What factors influence the quality of governance institutions? An Asian perspective, "Journal of Asian Economics", 70, https://doi.org/10 $.1016 /$ j.asieco.2020.101238

Hammami, S. (2019), Foreign direct investment inflows, intellectual property rights and economic freedom: Empirical evidence from middle-and low-income countries, "African Journal of Science, Technology, Innovation and Development”, 11 (7), pp. 861871, https://doi.org/10.1080/20421338.2019.1587909

Jabri, A., Brahim, M. (2015), Institutional Determinants of Foreign Direct Investment in MENA Region: Panel Co-Integration Analysis, "Journal of Applied Business Research", 31 (5), pp. 2001-2012, https://doi.org/10.19030/jabr.v31i5.9417

James, G., Witten, D., Hastie, T., Tibshirani, R. (2014), An Introduction to Statistical Learning with Applications in $R$, Springer, New York-Heidelberg-Dordrecht-London, https://doi.org/10.1007/978-1-4614-7138-7

Jurčić, L., Franc, S., Barišić, A. (2020), Impact of Institutional Quality on Foreign Direct Investment Inflow: Evidence from Croatia, "Business Systems Research", 11 (1), pp. 44-58, https://doi.org/10.2478/bsrj-2020-0004

Kunčič, A. (2014), Institutional quality dataset, "Journal of Institutional Economics", 10 (1), pp. 135-161, https://doi.org/10.1017/S1744137413000192

Kurul, Z., Yalta, A.Y. (2017), Relationship between Institutional Factors and FDI Flows in Developing Countries: New Evidence from Dynamic Panel Estimation, "Economies", 5 (2), https://doi.org/10.3390/economies5020017

Lasek, M. (2002), Data mining. Zastosowania w analizach i ocenach klientów bankowych, Biblioteka Menedżera i Bankowca, Warszawa.

Lehne, J., Mo, J., Plekhanov, A. (2014), What determines the quality of economic institutions? Cross-country evidence, "European Bank for Reconstruction and Development Working Paper”, No. 171, https://doi.org/10.2139/ssrn.3121122 
Madani, S., Nobakht, M. (2014), Political Regimes and FDI Inflows: Empirical Evidence from Upper Middle Income Countries, "Journal of Finance and Economics", 2 (3), pp. 75-82, https://doi.org/10.12691/jfe-2-3-4

Madr, M., Kouba, L. (2015), Does the Political Environment Affect Inflows of Foreign Direct Investment? Evidence from Emerging Markets, "Acta Universitatis Agriculturae et Silviculturae Mendelianae Brunensis”, 63 (6), pp. 2017-2026, https://doi.org /10.11118/201563062017

Miningou, E.W., Tapsoba, S.J. (2017), Education Systems and Foreign Direct Investment: Does External Efficiency Matter?, "IMF Working Paper", no. WP/17/79, https://doi .org/10.5089/9781475590234.001

Moon, Ch. (2019), Political institutions and FDI inflows in autocratic countries, "Democratization", 26 (7), pp. 1256-1277.

Nielsen, B.B., Asmussen, C.G., Weatherall, C. (2017), The Location Choice of Foreign Direct Investments: Empirical Evidence and Methodological Challenges, "Journal of World Business", 52 (1), pp. 62-82, https://doi.org/10.1016/j.jwb.2016.10.006

O’Meara, G. (2015), Examining the Determinants of Foreign Direct Investment, "Undergraduate Economic Review”, 11 (1), https:/digitalcommons.iwu.edu/uer/vol11 /iss1/13/ (accessed: 1.12.2020).

Peres, M., Ameer, W., Xu, H. (2018), The impact of institutional quality on foreign direct investment inflows: evidence for developed and developing countries, "Economic Research - Ekonomska Istraživanja”, 31 (1), pp. 626-644, https://doi.org/10.1080 /1331677X.2018.1438906

Rodriguez-Pose, A. (2013), Do Institutions Matter for Regional Development?, "Regional Studies”, 47 (7), pp. 1034-1047. https://doi.org/10.1080/00343404.2012.748978

Sabir, S., Rafique, A., Abbas, K. (2019), Institutions and FDI: evidence from developed and developing countries, "Financial Innovation", 5 (1), pp. 1-20. https://doi.org/10 .1186/s40854-019-0123-7

Sedik, W.M. (2012), The Impact of Country Risk and New Institutional Economics on Foreign Direct Investment: A Panel Data Analysis for Middle East and North Africa Region, The $16^{\text {th }}$ Meeting of the International Society for New Institutional Economics, University of Southern California, Los Angeles.

Seyoum, B., Manyak, T.G. (2009), The impact of public and private sector transparency on foreign direct investment in developing countries, "Critical Perspectives on International Business", 5 (3), pp. 187-206, https://doi.org/10.1108/17422040910974686

Stachowiak, K. (2007), Instytucjonalne uwarunkowania bezpośrednich inwestycji zagranicznych w Polsce, Bogucki Wydawnictwo Naukowe, Poznań.

Subasat, T., Bellos, S. (2011), Economic freedom and foreign direct investment in Latin America: a panel gravity model approach, "Economics Bulletin", 31 (3), pp. 151-156, https://doi.org/10.5539/jms.v3n4p151

Tan, P.N., Steinbach, M., Kumar, V. (2006), Introduction to Data Mining, Pearson Addison-Wesley, Boston.

Tun, Y.-L., Azman-Saini, W., Law, S.-H. (2012), International evidence on the link between foreign direct investment and institutional quality, "Engineering Economics", 23 (4), pp. 379-386, https://doi.org/10.5755/j01.ee.23.4.2569 
Türedi, S. (2018), The effect of corruption and country risk on FDI inflows: empirical evidence from developing countries, "International Journal of Economic \& Administrative Studies”, 21, pp. 151-172, https://doi.org/10.18092/ulikidince.370653

Ullah, I., Khan, M.A. (2017), Institutional quality and foreign direct investment inflows: evidence from Asian countries, "Journal of Economic Studies", 44 (6), pp. 1030-1050, https://doi.org/10.1108/JES-10-2016-0215

UNCTAD (2019), World Investment Report, Geneva.

Walsh, J.P., Yu, J. (2010), Determinants of Foreign Direct Investment: A Sectoral and Institutional Approach, "IMF Working Paper: Asia Pacific Department", no. WP/10/187, https://doi.org/10.5089/9781455202218.001

Yeboua, K. (2020), Foreign Direct Investment and Economic Growth in Africa: New Empirical Approach on the Role of Institutional Development, "Journal of African Business”, 22 (3), pp. 361-378, https://doi.org/10.1080/15228916.2020.1770040

\section{Jakość instytucjonalna i jej wpływ na zagraniczne inwestycje bezpośrednie: przykład państw członkowskich Unii Europejskiej}

Celem artykułu jest ocena jakości instytucjonalnej w 28 państwach członkowskich UE oraz próba oceny zależności pomiędzy jakością instytucjonalną a poziomem napływu zagranicznych inwestycji bezpośrednich (ZIB). Opracowanie ma następującą strukturę. Po pierwsze, dokonaliśmy przeglądu badań poświęconych związkom między jakością instytucjonalną a atrakcyjnością inwestycyjną. Następnie omówiliśmy napływ ZIB do krajów UE i wybraliśmy zmienne diagnostyczne, które posłużyły za podstawę do dalszej analizy. W tym celu posłużyliśmy się miernikami Globalnego Indeksu Konkurencyjności. W kolejnym etapie wykorzystując rankingi i metody statystyczne podzieliliśmy państwa członkowskie UE na grupy o zbliżonym poziomie jakości instytucjonalnej. Następnie zbadaliśmy zależności między podobnymi do siebie grupami krajów oraz grupami państw uszeregowanych według wartości napływu ZIB jako \% PKB.

Badanie wykazało, że państwa członkowskie UE różnią się wyraźnie pod względem jakości instytucjonalnej. Wyniki analiz statystycznych dały podstawę do pozytywnej weryfikacji hipotezy o pozytywnym związku między poziomem jakości instytucjonalnej a atrakcyjnością inwestycyjną.

Słowa kluczowe: ZIB, instytucje, jakość instytucjonalna, hierarchiczna analiza skupień, państwa członkowskie Unii Europejskiej 


\section{Appendix}

Table A1. Descriptions and names institutional quality indicators

\begin{tabular}{|c|c|c|}
\hline $\begin{array}{l}\text { Name } \\
\text { of variable }\end{array}$ & Description & Scale \\
\hline $\begin{array}{l}\text { Business costs } \\
\text { of organized } \\
\text { crime }\end{array}$ & $\begin{array}{l}\text { Response to the survey question "In your country, } \\
\text { to what extent does organized crime (mafia-oriented } \\
\text { racketeering, extortion) impose costs on businesses?" }\end{array}$ & $\begin{array}{l}{[1=\text { to } \text { a great extent, im- }} \\
\text { poses huge costs; } 7=\text { not } \\
\text { at all, imposes no costs] }\end{array}$ \\
\hline Homicide rate & Number of intentional homicides. & per 100,000 population \\
\hline $\begin{array}{l}\text { Terrorism inci- } \\
\text { dence }\end{array}$ & Assesses the frequency and severity of terror attacks. & $\begin{array}{l}\text { The scale ranges from } \\
0 \text { (highest incidence) } \\
\text { to } 100 \text { (no incidence) }\end{array}$ \\
\hline $\begin{array}{l}\text { Reliability } \\
\text { of police ser- } \\
\text { vices }\end{array}$ & $\begin{array}{l}\text { Response to the survey question "In your country, } \\
\text { to what extent can police services be relied upon } \\
\text { to enforce law and order?" }\end{array}$ & $\begin{array}{l}{[1=\text { not at all; }} \\
7=\text { to a great extent }]\end{array}$ \\
\hline Social capital & $\begin{array}{l}\text { Score on the Social Capital pillar of the Legatum Pros- } \\
\text { perity Index }{ }^{\mathrm{TM}} \text {, which assesses social cohesion and en- } \\
\text { gagement, community and family networks, and po- } \\
\text { litical participation and institutional trust. }\end{array}$ & $\begin{array}{l}\text { The scale ranges from } \\
0 \text { (low) to } 100 \text { (high) }\end{array}$ \\
\hline $\begin{array}{l}\text { Budget trans- } \\
\text { parency }\end{array}$ & $\begin{array}{l}\text { Assesses the amount and timeliness of budget infor- } \\
\text { mation that governments make publicly available. }\end{array}$ & $\begin{array}{l}\text { The scale ranges from } \\
0 \text { (low) to } 100 \text { (best) }\end{array}$ \\
\hline $\begin{array}{l}\text { Judicial inde- } \\
\text { pendence }\end{array}$ & $\begin{array}{l}\text { Response to the survey question "In your country, } \\
\text { how independent is the judicial system from influenc- } \\
\text { es of the government, individuals, or companies?" }\end{array}$ & $\begin{array}{l}\text { [1 = not independent } \\
\text { at all; } 7=\text { entirely inde- } \\
\text { pendent] }\end{array}$ \\
\hline $\begin{array}{l}\text { Efficiency of le- } \\
\text { gal framework } \\
\text { in challenging } \\
\text { regulations }\end{array}$ & $\begin{array}{l}\text { Response to the survey question "In your country, } \\
\text { how easy is it for private businesses to challenge gov- } \\
\text { ernment actions and/or regulations through the legal } \\
\text { system?" }\end{array}$ & $\begin{array}{l}\text { [1 = extremely difficult; } \\
7 \text { = extremely easy] }\end{array}$ \\
\hline $\begin{array}{l}\text { Freedom of the } \\
\text { press }\end{array}$ & $\begin{array}{l}\text { Score on the World Press Freedom Index, which } \\
\text { measures the level of freedom available to journalists. }\end{array}$ & $\begin{array}{l}\text { The scale ranges from } 0 \\
\text { (good) to } 100 \text { (very bad) }\end{array}$ \\
\hline $\begin{array}{l}\text { Burden of gov- } \\
\text { ernment regu- } \\
\text { lation }\end{array}$ & $\begin{array}{l}\text { Response to the survey question "In your country, } \\
\text { how burdensome is it for companies to comply with } \\
\text { public administration's requirements (e.g., permits, } \\
\text { regulations, reporting)?" }\end{array}$ & $\begin{array}{l}\text { [1 = extremely burden- } \\
\text { some; } 7 \text { = not burden- } \\
\text { some at all] }\end{array}$ \\
\hline $\begin{array}{l}\text { Efficiency of le- } \\
\text { gal framework } \\
\text { in settling dis- } \\
\text { putes }\end{array}$ & $\begin{array}{l}\text { Response to the survey question "In your country, } \\
\text { how efficient are the legal and judicial systems for } \\
\text { companies in settling disputes?" }\end{array}$ & $\begin{array}{l}\text { [1 = extremely ineffi- } \\
\text { cient; } 7=\text { extremely ef- } \\
\text { ficient }]\end{array}$ \\
\hline E-Participation & $\begin{array}{l}\text { Score on the E-Participation Index, which assesses } \\
\text { the use of online services to facilitate the provision } \\
\text { of information by governments to citizens. }\end{array}$ & $\begin{array}{l}\text { The scale ranges from } \\
0 \text { to } 1 \text { (best) }\end{array}$ \\
\hline
\end{tabular}


Institutional Quality and its Impact on FDI Inflow: Evidence from the EU Member States

\begin{tabular}{|c|c|c|}
\hline $\begin{array}{l}\text { Name } \\
\text { of variable }\end{array}$ & Description & Scale \\
\hline $\begin{array}{l}\text { Future orienta- } \\
\text { tion of govern- } \\
\text { ment }\end{array}$ & $\begin{array}{l}\text { Average of the responses to the following four Ex- } \\
\text { ecutive Opinion Survey questions: 1) "In your coun- } \\
\text { try, how fast is the legal framework of your country } \\
\text { in adapting to digital business models (e.g., e-com- } \\
\text { merce, sharing economy, fintech, etc.)?"; 2) "In your } \\
\text { country, to what extent does the government ensure } \\
\text { a stable policy environment for doing business?"; } \\
\text { 3) "In your country, to what extent does the govern- } \\
\text { ment respond effectively to change (e.g., techno- } \\
\text { logical changes, societal and demographic trends, } \\
\text { security and economic challenges)?"; and 4) "In your } \\
\text { country, to what extent does the government have } \\
\text { a long-term vision in place?". }\end{array}$ & $\begin{array}{l}\text { [1 = not fast at all; } \\
7=\text { very fast] } \\
\text { For the last three ques- } \\
\text { tions, the answer ranges } \\
\text { from } 1 \text { (not at all) to } \\
7 \text { (to a great extent). }\end{array}$ \\
\hline $\begin{array}{l}\text { Incidence } \\
\text { of corruption }\end{array}$ & $\begin{array}{l}\text { Score on the Corruption Perceptions Index, which } \\
\text { measures perceptions of corruption in the public sec- } \\
\text { tor. This is a composite indicator. }\end{array}$ & $\begin{array}{l}\text { The scale ranges from } \\
0 \text { (highly corrupt) } \\
\text { to } 100 \text { (very clean) }\end{array}$ \\
\hline Property rights & $\begin{array}{l}\text { Response to the survey question "In your country, } \\
\text { to what extent are property rights, including financial } \\
\text { assets, protected?" }\end{array}$ & $\begin{array}{l}{[1=\text { not at all; }} \\
7=\text { to a great extent }]\end{array}$ \\
\hline $\begin{array}{l}\text { Intellectual } \\
\text { property pro- } \\
\text { tection }\end{array}$ & $\begin{array}{l}\text { Response to the survey question "In your country, } \\
\text { to what extent is intellectual property protected?" }\end{array}$ & $\begin{array}{l}{[1=\text { not at all; }} \\
7=\text { to a great extent }]\end{array}$ \\
\hline $\begin{array}{l}\text { Quality of land } \\
\text { administration }\end{array}$ & $\begin{array}{l}\text { Score on the quality of land administration index, } \\
\text { which assesses the reliability of infrastructure, trans- } \\
\text { parency of information, geographic coverage, land } \\
\text { dispute resolution, and equal access to property } \\
\text { rights. }\end{array}$ & $\begin{array}{l}\text { The scale ranges from } \\
0 \text { to } 30 \text { (best) }\end{array}$ \\
\hline $\begin{array}{l}\text { Strength of au- } \\
\text { diting and re- } \\
\text { porting stand- } \\
\text { ards }\end{array}$ & $\begin{array}{l}\text { Response to the survey question "In your coun- } \\
\text { try, how strong are financial auditing and reporting } \\
\text { standards?" }\end{array}$ & $\begin{array}{l}\text { [1 = extremely weak; } \\
7 \text { = extremely strong] }\end{array}$ \\
\hline $\begin{array}{l}\text { Conflict of in- } \\
\text { terest regula- } \\
\text { tion }\end{array}$ & $\begin{array}{l}\text { Score on the extent of conflict of interest regulation } \\
\text { index, which measures the protection of sharehold- } \\
\text { ers against directors' misuse of corporate assets for } \\
\text { personal gain. }\end{array}$ & $\begin{array}{l}\text { The scale ranges from } \\
0 \text { to } 10 \text { (best) }\end{array}$ \\
\hline $\begin{array}{l}\text { Shareholder } \\
\text { governance }\end{array}$ & $\begin{array}{l}\text { Score on the extent of shareholder governance index, } \\
\text { which measures shareholders' rights in corporate } \\
\text { governance. }\end{array}$ & $\begin{array}{l}\text { The scale ranges from } \\
0 \text { to } 10 \text { (best) }\end{array}$ \\
\hline
\end{tabular}

Source: own elaboration based on $\mathrm{GCl}$. 
Bogusława Dobrowolska, Tomasz Dorożyński, Anetta Kuna-Marszałek

Table A2. Mean value of institutional quality variables of the EU Member States

in the years 2018-2019 by groups distinguished based on the dendrogram

\begin{tabular}{|c|c|c|c|c|c|}
\hline \multirow{2}{*}{ Country Group (1-5) } & Group 1 & Group 2 & Group 3 & \begin{tabular}{|l|} 
Group 4 \\
\end{tabular} & Group 5 \\
\hline & Mean & Mean & Mean & Mean & Mean \\
\hline Organized crime (1-7) & 5.37 & 5.93 & 4.71 & 5.54 & 5.23 \\
\hline Homicides per 100,000 population & 0.96 & 1.52 & 0.91 & 0.77 & 2.18 \\
\hline $\begin{array}{l}\text { Terrorism incidence ( } 0 \text { = very high; } \\
100=\text { no incidence) }\end{array}$ & 97.48 & 97.69 & 91.09 & 99.65 & 99.93 \\
\hline Reliability of police services (1-7) & 5.69 & 6.04 & 4.47 & 5.35 & 4.49 \\
\hline Social capital (0-100, high) & 60.84 & 59.22 & 49.36 & 59.84 & 48.82 \\
\hline Open Budget Data score $(0-100$, best $)$ & 70.51 & 67.31 & 56.41 & 56.39 & 57.69 \\
\hline Judicial independence (1-7) & 5.79 & 6.09 & 3.57 & 4.03 & 3.88 \\
\hline $\begin{array}{l}\text { Efficiency of legal framework in challenging } \\
\text { regulations (1-7) }\end{array}$ & 4.51 & 4.72 & 2.54 & 3.16 & 2.72 \\
\hline $\begin{array}{l}\text { World Press Freedom Index }(0=\text { most free; } \\
100=\text { least free })\end{array}$ & 86.40 & 85.31 & 73.15 & 77.54 & 78.41 \\
\hline Burden of government regulation (1-7) & 3.88 & 4.03 & 2.59 & 3.06 & 2.96 \\
\hline $\begin{array}{l}\text { Efficiency of legal framework to settle } \\
\text { disputes (1-7) }\end{array}$ & 5.10 & 4.90 & 2.50 & 3.44 & 3.21 \\
\hline E-Participation Index (0-1, best) & 0.88 & 0.85 & 0.82 & 0.85 & 0.66 \\
\hline Future orientation of government (1-7) & 4.61 & 4.62 & 2.87 & 3.81 & 3.24 \\
\hline $\begin{array}{l}\text { Corruption Perception Index }(0=\text { highly } \\
\text { corrupt; } 100=\text { very clean })\end{array}$ & 80.00 & 78.17 & 52.17 & 58.00 & 53.07 \\
\hline Property rights (1-7) & 5.81 & 6.02 & 4.01 & 4.71 & 4.35 \\
\hline Intellectual property protection (1-7) & 5.83 & 6.02 & 4.14 & 4.73 & 4.47 \\
\hline $\begin{array}{l}\text { Quality of land administration index } \\
(0-30 \text {, best })\end{array}$ & 24.92 & 24.58 & 18.83 & 19.50 & 24.00 \\
\hline $\begin{array}{l}\text { Strength of auditing and accounting standards } \\
(1-7)\end{array}$ & 5.89 & 5.86 & 4.30 & 5.08 & 4.86 \\
\hline $\begin{array}{l}\text { Extent of conflict of interest regulation } \\
(0-10, \text { best })\end{array}$ & 5.68 & 6.67 & 5.90 & 6.43 & 5.53 \\
\hline Extent of shareholder governance $(0-10$, best $)$ & 7.23 & 5.68 & 6.82 & 7.03 & 6.24 \\
\hline
\end{tabular}

Source: own elaboration (calculations were made in PS IMAGO).

\begin{tabular}{|l|l|}
\hline CC by the author, licensee Łódź University - Łódź University Press, \\
tódź, Poland. This article is an open access article distributed under \\
the terms and conditions of the Creative Commons Attribution \\
license CC-BY-NC-ND 4.0 \\
(https://creativecommons.org/licenses/by-nc-nd/4.0/) \\
Received: 2021-01-04. Verified: 2021-07-12. Accepted: 2021-08-11.
\end{tabular}

